Article

\title{
Executive functions and cannabis use in adolescents
}

\author{
Alessandro Frolli ${ }^{1,2 *}$, Maria Carla Ricci², Antonella Cavallaro², Agnese Lombardi², Grazia Maria Giovanna \\ Pastorino $^{3}$, Francesca Felicia Operto ${ }^{3 *}$ \\ ${ }^{1}$ University of International Studies of Rome, Rome, Italy \\ ${ }^{2}$ FINDS- Italian Neuroscience and Developmental Disorders Foundation, Caserta, Italy \\ ${ }^{3}$ Child Neuropsychiatry Unit, Department of Medicine, Surgery and Odontostomatology, University of Salerno, \\ Salerno, Italy
}

\section{*Corresponding Author1: Operto Francesca Felicia \\ Child Neuropsychiatry Unit, Department of Medicine, Surgery and Odontostomatology, University of Salerno, 84081, Baronissi (SA), Italy; +39 089672578; +39 3471735041; e-mail: opertofrancesca@gmail.com}

\author{
*Corresponding Author2: Frolli Alessandro \\ alessandro.frolli@unint.eu - 3474910178 \\ Disability Research Centre of University of International Studies in Rome \\ Via Cristoforo Colombo, 200, Roma - Italy
}

\begin{abstract}
The developmental phase of adolescence is characterized by a multitude of neurocognitive and psychosocial changes and is therefore considered one of the most critical developmental periods of life. Experimentation on the use of substances often begins in adolescence and so does the addiction process. Most research in human subjects shows that chronic cannabis abuse is the cause of the impairment of some cognitive functions, affecting the performance on divided attention, verbal memory and working memory. In this study, we wanted to investigate how the abuse of cannabis (chronic, occasional and absence use) can influence global cognitive functioning, also through executive functions. From the statistical analyzes of our study, it emerges that the group of subjects who use chronic cannabis (group 1) has a significant drop in working memory tasks compared to the group that does not use it (group 3). In addition, the goal of future studies by our group is to verify the permanent alteration of cognitive processes affected through revaluations with calendar follow-up (controlled).
\end{abstract}

Keywords Adolescents, Cannabis, Cognition, Working Memory, Executive Functions

\section{Introduction}

The developmental phase of adolescence is characterized by a multitude of neurocognitive and psychosocial changes and is therefore considered one of the most critical developmental periods of life (Giedd, 2015). As matter of fact, from the age of 12, a new process of brain maturation begins the adolescencial changes, through the phases of proliferation, migration and differentiation, synaptogenesis and pruning, and the whole process will lead to a global remodeling of the brain. Cognitive functions and processes that involve the frontal and prefrontal cortex, fully develop mainly during adolescence, where, the higher cognitive functions reach their final maturity (Gogtay et al., 2004). In this phase the substances of abuse can interfere considerably. Consumption of substances often begins in adolescence and so does the addiction process (Volkow et al., 2016). For example, over $90 \%$ of people who are addicted today started using various substances before they were 18 years old (Public Health Agency of Canada, 2018). Cannabis is the most frequently used illegal substance in the world and its usage is expected to increase with recent changes in its legal status. Most research in human subjects shows that chronic cannabis abuse causes impairment of 
some cognitive functions, affecting performance on divided attention, verbal memory, and working memory (Broyd et al., 2016; Hindocha et al., 2017; Lundqvist et al., 2005; Solowij et al., 2002; Bull et al., 2002; Ilan et al., 2004). In particular, working memory suffers the effects of substance abuse, especially because its impairment also affects other cognitive functions. According to some studies, this impairment causes below-average academic performance (Grant et al., 2003), declarative memory deficit (Grant et al., 2012) and persistent decline in global cognitive functioning (Meier et al., 2012). Studies showing that impairment persists in adulthood are particularly worrying, (Alloway et al., 2010; Ullman et al., 2014) and with greater severity among chronic cannabis users (Cousijn et al., 2014; Solowij et al., 2008). Pope \&Yurgelun (1996), document one of the fewer studies comparing adolescents with a chronic and a occasional cannabis consumption behaviour. In more recent studies, Pope et al., (2001) undergo to cannabis administration and after a 19 hours time frame of abstinence, detected an impairment affecting memory capabilities, particularly attention and executive functions. Successively Pope et al., (2002) widened the sample using more specific and sensitive tests founding a greater specific impairment of visual-spatial memory in chronic consumers than in occasional ones. Chronic cannabis usage in adolescence may cause permanent changes to neuronal circuits in specific brain areas and such changes may increase the likelihood of developing psychiatric disorders in adulthood (Gardner \& Steinberg, 2005; Rubino et al., 2011). Evidence also suggests that individuals who start consuming cannabis at an early age may be more vulnerable to long-term neuropsychological deficits than individuals who started using it later (Porath-Waller, 2009). In this study investigated how cannabis abuse (chronic and occasional consumption, as well as absence) can influence global cognitive functioning, also through executive functions. The methodology used was the administration of WISC-IV (Orsini, Pezzuti and Picone, 2012) in two groups of adolescents with occasional and chronic cannabis usage. In particular, working memory indexes (WMI), processing speed (PSI) and intellectual quotient (IQ) were investigated by comparing scores with a control group that did not consume substances, to understand the impairment magnitude that the abuse may cause to cognitive functions. We also performed a neuropsychological study through the administration of the BVN Battery 12-18 to investigate whether planning skills and memory capacities (visuo-spatial) were affected by the consumption of cannabinoid substances.

\section{Materials and Methods}

\subsection{Participants}

The study included subjects from 10 secondary grade schools in the province of Naples, aged between 15 and 16. The study is a collaboration of, the Italian Foundation for Neuroscience and Development Disorders (FINDS) and the Neuropsychiatry centerof the University of Salerno, with the Regional School Office of the region Campania (USR). The selected subjectsundergo to a preliminary questionnaire to report adolescents habits with respect to the usage of cannabinoids. The questionnaire consists of 6 questions, where 5 required a 5-item Likert-scale response and a semi-open question to quantify the use of cannabinoids. The open-ended question played a crucial role in order to stratify the subjects in 3 groups: group 1 comprising of 46 chronic consumers of cannabis (at least 4 times a week in the last year), group 2 with 46 occasionally consumers subjects (about once every two weeks in the last year) and a group 3 (control) including 46 selected subjects of non consumers. All the groups undertake the administration of WISC IV within the school setting, anonymously, by qualified psychologists belonging to the two clinics of reference (FINDS and University of Salerno). Afterwards, the Corsi and ToL subtests were administered by BVN 12-18 (Gugliotta et al., 2009) [Table 1].

\begin{tabular}{cccc}
\hline & Group 1 & Group2 & Group 3 \\
\hline $\begin{array}{l}\text { Age in years } \\
(\text { mean } \pm \text { SD) }\end{array}$ & $15.3 \pm 0.23$ & $15.3 \pm 0.11$ & $15.4 \pm 0.11$ \\
\hline
\end{tabular}


(Male/Femmale)

Table 1. Sample characteristics; Mage=mean age; $\mathrm{SD}=$ standard deviation; $\mathrm{M}=$ male; $F=F e m a l e$

\subsection{Procedures and tasks}

The protocol used consists of: a specifically constructed questionnaire, investigating the frequency of cannabinoid consumption, the WISC-IV (Orsini, Pezzuti and Picone, 2012), and the BVN 12-18 (Gugliotta et al., 2009).

Questionnaire: The questionnaire included 5 closed questions on the following points: possible consumption of cannabinoids (regular or occasional), possible consumption of other substances (regular or occasional), possible usage of alcohol (regular or occasion), tendency to use cannabinoids and other substances or alcohol (individual and group). The answers were based on a 5-point Likert scale ranging from one (strongly agree) to five (strongly disagree). For affirmative answers on the usage of cannabinoids, the weekly frequency was also investigated through an open question.

WISC-IV: clinical and diagnostic tool that allows to evaluate the intellectual abilities of children aged from 6 to 16 years. It consists of 15 tests (10 main and 5 additional) divided into 4 indices. The 10 main tests consist of: drawing with cubes (BD), similarities (SI), digits span (DS), illustrated concepts $(\mathrm{PCN})$, cipher $(\mathrm{CD})$, vocabulary $(\mathrm{VC})$, rearrangement letters-numbers $(\mathrm{LN})$, reasoning with matrices (MR), comprehension (CO), search for symbols (SS). The tests are divided in 4 indices: perceptive reasoning index (PRI), which includes $\mathrm{BD}, \mathrm{PCN}$ and $\mathrm{MR}$, verbal comprehension index (VCI), which includes SI, VC and CO, working memory index (WMI) that includes DS and LN, and processing speed index (PSI) that includes CD and SS.

BVN 12-18: Test battery for neuropsychological evaluation allowing to identify individual disorders in specific areas and to define a general profile of mnemonic, praxis, visuospatial, perceptive, attentive, linguistic, executive skills, etc., useful for further analysis after an initial diagnostic classification. In particular, the ToL (Tower of London) subtest, allows to evaluate higher executive functions such as planning and problem-solving skills. The Corsi subtest allows to evaluate the span of visuo-spatial memory, namely, the amount of visuo-spatial information that can be retained in short-term memory (MBT).

\subsection{Procedures}

The students sample population undergo to the questionnaire in order to investigate the frequency of the substances consumption. From the emerged results, we divided the sample into three groups: group 1, made of chronic users of cannabinoids, group 2, including occasional users, and group 3 of non-consumers. The third group was randomly obtained among those who emerged as being non users of substances, after undertaking the questionnaire. All groups then performed the WISC-IV, in its Italian standardization. Scores were calculated from the 4 indices: the perceptive reasoning index (PRI), which includes $\mathrm{BD}, \mathrm{PCN}$ and $\mathrm{MR}$, the verbal comprehension index (VCI), which includes SI, VC and CO, the working memory index (WMI) which includes DS and LN, and the processing speed index (PSI) which includes CD and SS. Individual task scores were analyzed, in particular the main indices (VCI, PRI, WMI, PSI and IQ). Our research hypothesis suggests that the chronical cannabis users (group 1) has a fall (difficulty) relevant to the working memory (WMI) and processing speed (PSI) tasks, with consequent impact on the IQ, and this is consistent with previous literature (Fried, Watkinson and Grey, 2005). We then performed the subtests of the BVN 12-18 for an in-depth study of memory skills (visuo-spatial) (Test of Corsi) and planning (subtest of the Towers of London). Our findings report that the group of subjects who compulsively consume cannabis (group 1) has a significant drop in planning (ToL) and visuo-spatial memory (Corsi) tasks, compared to the control group (group 3). 


\section{Results}

Data analysis was carried out using SPSS 25.0 statistical survey software. Significance was accepted at the $5 \%$ level $(\alpha<0.05)$. We compared the weighted scores of the indices (VCI, PRI, WMI, PSI, IQ) emerged from the WISC-IV through the use of the Student's T test, a parametric statistical test that can be used when the two groups in comparison are independent of each other. Specifically, we used the student $\mathrm{t}$-test for unpaired samples, in order to make comparisons between groups, with two-tailed significance. The comparison between group 1 and group 3 showed significant differences to the WMI indices $(t=-13,38 ; \mathrm{p}<0.05)$, PSI $(t=-4,89 ; \mathrm{p}<0.05)$ and IQ $(t=-9,56 ; \mathrm{p}<0.05)$. These results show that chronic use of cannabinoids has, compared to those who do not make any use of cannabinoids, a significant effect on the functioning of working memory, on the speed of information processing, and also affects the IQ. Slightly significant is the impact on the PRI $(t=-3,02$; $\mathrm{p}<0.05$ ), while the impact on the VCI is not significant. From the comparison between group 1 and group 2, significant differences emerged at the indices WMI $(t=-11,37 ; p<0.05)$, PSI $(t=-4,75 ; p<0.05)$ and IQ $(t=-7,31 ; p<0.05)$. These results suggest that a chronic use of cannabinoids, compared to those who use it less frequently, can affect the working memory and speed of information processing skills, thus impacting the global cognitive functioning, represented by the IQ index. We subsequently compared the scores that emerged at the ToL subtest between group 1 and group 2 and significant differences emerged $(t=-3,56 ; p<0.05)$. These results demonstrate that chronic use of cannabinoids has a significant impact on planning skills compared to those who use it less regularly. Significant differences also emerged in the comparison between group 1 and group $3(t=-4,52 ; p$ $<0.05)$, showing that chronic consumers of cannabinoids have significant difficulties in planning skills compared to non-consumers. There were no significant differences between group 2 and group 3. Finally, we compared the performance at the subtest of Corsi, between group 1 and group 2 and found significant differences $(t=-7,64 ; \mathrm{p}<0.05)$. These results indicate that chronic consumers of cannabinoids experience considerable difficulties in visuo-spatial memory skills. Significant differences also emerged between group 1 and group $3(t=-9,38 ; \mathrm{p}<0.05)$. These results show that chronic use of cannabinoids can affect visual-spatial memory skills, compared to those who do not use it. However, no significant differences were found between group 2 and group 3 (Table 2).

\begin{tabular}{ccccccc}
\hline & \multicolumn{2}{c}{ Group 1 } & \multicolumn{2}{c}{ Group 2 } & \multicolumn{2}{c}{ Group 3 } \\
\cline { 2 - 7 } & Mean & SD & Mean & SD & Mean & SD \\
\hline VCI & 98,83 & 4,33 & 101,26 & 5,51 & 101,65 & 5,77 \\
WMI & 83,17 & 6,11 & 96,22 & 4,58 & 97,85 & 4,80 \\
PSI & 92,24 & 5,78 & 98,37 & 5,82 & 98,30 & 5,39 \\
IQ & 89,17 & 6,51 & 99,91 & 6,39 & 101,83 & 7,40 \\
ToL & 7,30 & 0,94 & 8,07 & 1,06 & 8,35 & 1,21 \\
CORSI & 3,87 & 0,71 & 5,11 & 0,84 & 5,46 & 1,00 \\
\hline
\end{tabular}


Table 2. Comparison of WISC-IV indices between the three groups; VCI=Verbal Comprehension Index; PRI= Perceptual Reasoning Index; WMI= Working Memory Index; PSI=Parental Stress Index; $\mathrm{IQ}=$ Intelligence Quotient; ToL= Tower of London Test; CORSI= Corsi Test; M=mean; SD=standard deviation

To understand which differences are greater between the groups, we performed an ANOVA (Analysis of variance) with post hoc tests (Bonferroni). From these analysis it emerged that group 1 scores differ significantly from those of group 3 in the following indices: WMI $(-14,67 ; p<0,05)$, PSI $(-6,06 ; p<0,05)$ and IQ $(-12,65 ; p<0,05)$, demonstrating that chronic use of cannabinoid substances has a significant impact on the functioning of working memory, the speed of information processing, also impacting on IQ. The comparison between group 2 and group 3 scores showed no significant differences, while the comparison between group 1 and group 2 showed differences to the WMI indices $(-13,04 ; \mathrm{p}<0.05)$, PSI $(-6,13 ; \mathrm{p}<0.05)$ and IQ $(-10,73 ; \mathrm{p}<0.05)$, showing that the effects of chronic cannabis use on cognitive functioning are greater, while the occasional use of cannabis does not have a significant impact [Figure 1, 2,3].

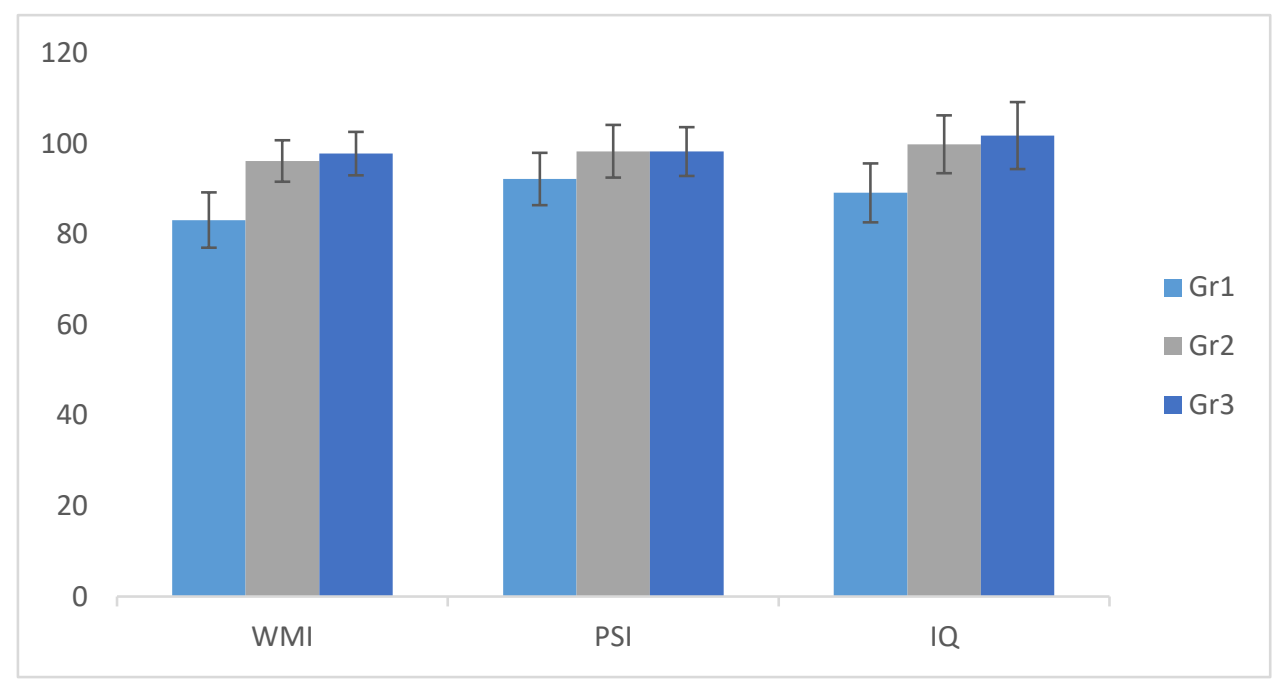

Figure 1 Comparison of indices WISC between the three groups; WMI= Working Memory Index; PSI=Parental Stress Index; IQ=Intelligence Quotient. Gr1=Group1; Gr2=Group2; Gr3=Group3. 


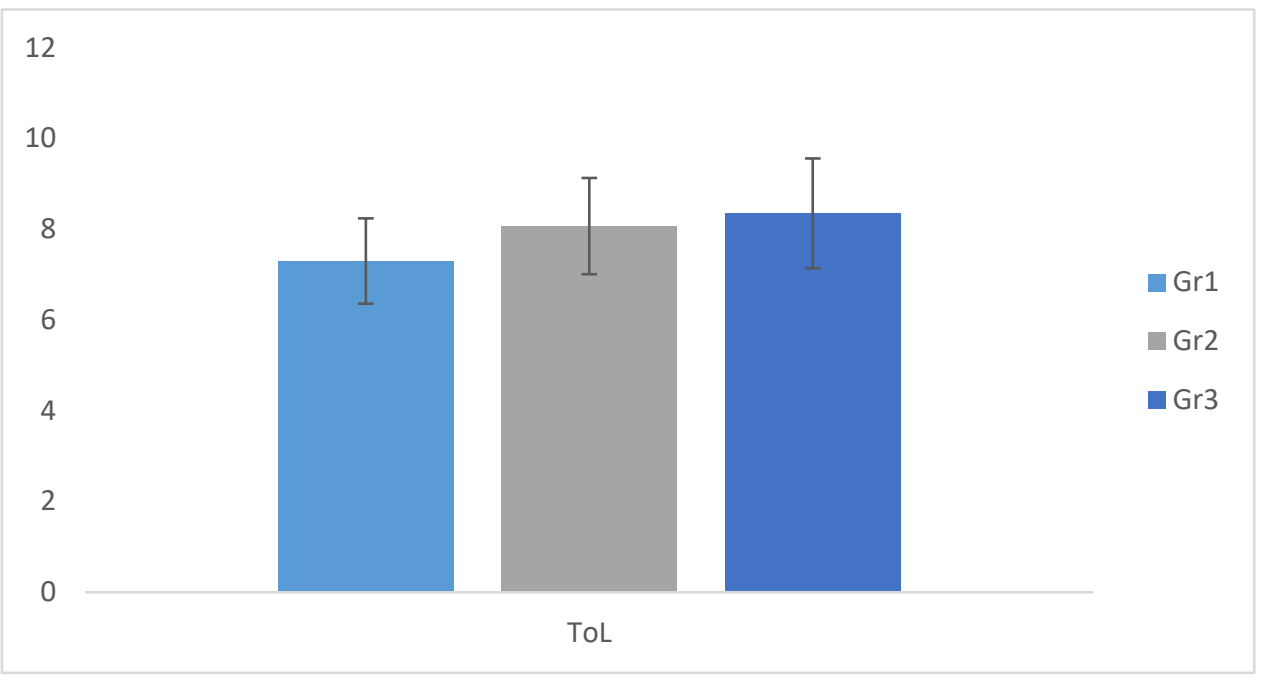

Figure 2. Comparison of ToL between the three groups; Tol=Tower of London; Gr1=Group1; Gr2=Group2; Gr3=Group3.

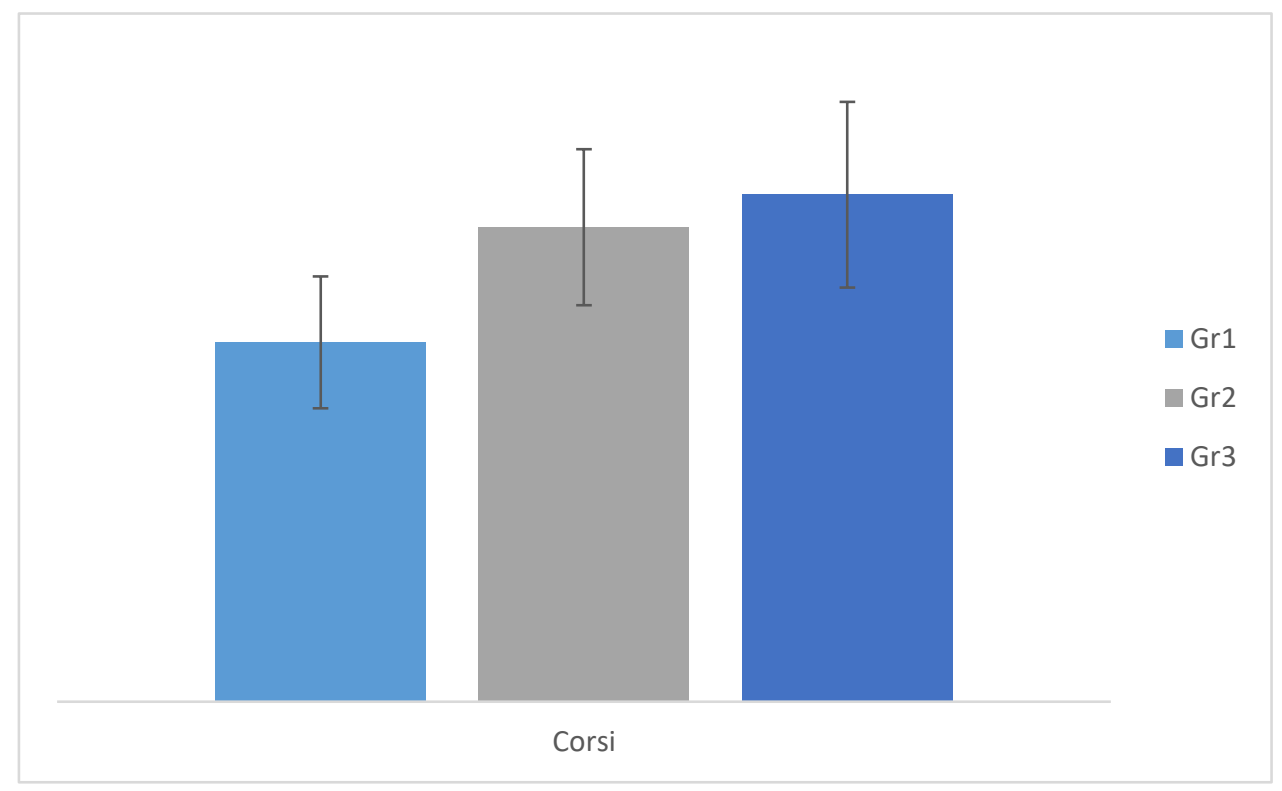

Figure 3. Comparison of Corsi Test between the three groups. Gr1=Group1; Gr2=Group2; Gr3=Group3.

\section{Discussion}

Several studies have shown high rates of substance abuse among young people; the greater ease with which such substances can be found is accompanied by an increasingly early use. Persistent cannabis consume before the age of 18 would cause permanent cognitive damage to intellectual, attentive and memory functions. Furthermore, stopping cannabinoids usage, does not seem to have the effect of restoring cognitive functions. The impairment of working memory is widely documented, especially in studies where the population is represented by adolescents who abuse cannabinoids. In these studies, in fact, scores were indicating a much lower performance to the working memory by comparing them with non-consumers subjects (Hoa et al., 2014). It was also found that changes of these cognitive process persist beyond the period of acute intoxication and are 
related to a variety of parameters: in fact, it has been shown that deficits increase as a function of frequency, the duration and age of cannabis use, but the precise parameters causing long-term deficits remain to be determined. Studies that have been conducted on cannabis users who have experienced a reasonably long withdrawal period suggest that working memory deficits may persist for some time after acute intoxication, but if the latter reflects the action of residues of substances resulting in chronic rather than sporadic usage, it remains to be determined (Solowij and Battisti R, 2008). The reason lies in the fact that before the age of 18 the brain is still in the organization and restructuring processes (the pruning phase is still in progress) and therefore more vulnerable to the damage caused by the consume of drugs. Other studies reported that cannabis consumprion also has an influence on cognitive functions, in particular they found a fall in tasks of attention, learning, working memory and speed of information processing (Fletcher et al., 1996, Pope \&Yurgelun-Todd 1996, Solowij et al., 2002). From the statistical analysis we performed in our study, it emerges that the group of subjects chronically consuming cannabis (group 1) shows a significant drop in working memory tasks compared to the group that does not use it (group 3). In addition, there is also a decrease in information processing speed and IQ tasks, showing a significant difference with the control group (group 3). The analysis of the scores within the group of occasional consumers of cannabis (group 2), shows that there is no decrease in working memory, processing speed, and intellectual quotient, regarding the control group (group 3). Finally, comparison between of group 1 and group 2, shows that there are significant falls to the working memory, to the processing speed and intellectual quotient of group 1 compared to group 2 . This shows that the use of substances at an early age has effects on cognitive functioning, on working memory and on processing speed, especially if the consumption is chronic. Therefore, the impact on cognitive processes, which emerged in previous studies (Rubino et al., 2008; Lisdahl\& Price, 2012), is a function of chronicity. Furthermore, with regard to the neuropsychological analysis carried out on our sample, the statistical analysis revealed that the group described as chronic consumers of cannabis (group 1) had a significant impairment of planning skills compared to the control group (group 3), thus noting an impact of the substance also on the executive functions, in particular those of planning. The impairment of the aforementioned functions was less minor as regards the group that made occasional use of the substances (group 2) compared to group 3. These data indicates that chronic use of cannabis significantly affects the functioning of higher cognitive processes such as precisely the executive functions, to a greater extent than the group that made no use (group 3). In addition, our analysis found that the group that made chronic use of cannabis (group 1) also had a greater drop in visuo-spatial material processing skills than the group that did not use cannabinoid substances (group 3). These data indicates that chronic cannabis use significantly compromises memory abilities, and to a significant extent those that allow the processing of visual-spatial material. Some research have noted deficits in learning, in mnestic functions, in working memory, in executive and attentive functions in adolescents who used chronic cannabis, and even after the interruption of the use of the substance, significant compromises remained (Millsaps et al., 1994). Also a more recent study, conducted by Morin et al., (2018) aimed to understand the relationship between cannabis use and cognitive development among adolescents at different levels of use (abstinence, occasional use and habitual use), noted that the use of cannabis in adolescence was associated with generally lower performance in working memory (WMI), perceptual reasoning (PRI) and processing speed (PSI). The results of this study suggest that individuals who start chronically using cannabis at an early age such as adolescence are vulnerable to cognitive deficits in working memory and in processing speed, and more specifically the overall cognitive functioning. Many clinical studies agree that the earlier the onset of cannabis use, the greater the risk of later developing psychiatric disorders or addiction to other substances from abuse (O'Shea M. et al., 2004;2006; Casey BJ. et al., 2008; Rubino et al., 2011). Furthermore, interactions between genes, environment and behavioural factors can influence the functioning of neurocognitive processes that occur when a substance dependence is present. Future studies should attempt to identify possible genetic predispositions contributing to neurocognitive abnormalities among adolescent chronic cannabis users. Therefore, more careful consideration of memory deficits associated with specific parameters 
of cannabis use and interactions with age, IQ, personality factors, genetics and neural substrates, including the endogenous cannabinoid system, could provide an explanation of the effects of cannabis on memory, global cognitive functioning and brain function and recovery potential through abstinence. Combined multidisciplinary research approaches, including cognitive process studies, neuroimaging studies, neurochemicals and genetics, promise much for future research in this field. In addition, the goal of future studies by our group is to verify the permanent alteration of cognitive processes affected through revaluations with calendar follow-up (controlled).

\section{Funding:}

The authors have no funding to disclose

\section{Conflicts of Interest}

The authors declare that they have no conflict of interest

\section{Compliance with Ethical Standards}

All procedures performed in studies involving human participants were in accordance with the ethical standards of the institutional research committee and with the 1964 Helsinki Declaration and its later amendments or comparable ethical standards

\section{References}

1. Alloway TP, Alloway RG. (2010). Investigating the predictive roles of working memory and IQ in academic attainment. J Exp Child Psychol; 106:20-29.

2. Bolla KI, Brown K, Eldreth D, Tate K, \& Cadet JL (2002). Dose-related neurocognitive effects of marijuana use. Neurology, 59(9), 1337-1343.

3. Broyd SJ, van Hell HH, Beale C, Yücel M, \&Solowij N (2016). Acute and Chronic Effects of Cannabinoids on Human Cognition-A Systematic Review. Biol Psychiatry, 79(7), 557-567. doi: 10.1016/j.biopsych.2015.12.002.

4. Casey BJ., Getz S. \& Galvan A. (2008), The adolescent brain. Dev Rev;28(1):62-77.

5. Cousijn J, Wiers RW, Ridderinkhof KR, van den Brink W, Veltman DJ, Goudriaan AE. (2014). Effect of baseline cannabis use and working- memory network function on changes in cannabis use in heavy cannabis users: a prospective fMRI study. Hum Brain Mapp; 35:2470-2482.

6. Fletcher JM., Page JB., Francis DJ., Copeland K., Naus MJ., Davis CM. et al. (1996), Cognitive correlates of long-term cannabis use in Costa Rican men. Arch Gen Psychiatry 1996; 53:1051-7.

7. Fried P. A., Watkinson B. \& Gray R. (2005), Neurocognitive consequences of marihuana - A comparison with pre-drug performance. Neurotoxicology and Teratology, 27(2), 231-239. doi:10.1016/ j.ntt.2004.11.003.

8. Gardner M. \& Steinberg L. (2005), Peer influence on risk taking, risk preference, and risky decision making in adolescence and adulthood: An experimental study. Developmental Psychology; 41:625-635.

9. Giedd J. N. (2015). Adolescent neuroscience of addiction: a new era. Dev. Cogn. Neurosci. 16, $192-193$. 10.1016/j.dcn.2015.11.002.

10. Gogtay N., Giedd JN., Lusk L., Hayashi KM., Greenstein D., Vaituzis AC. et al. (2004), Dynamic mapping of human cortical development during childhood through early adulthood. Proc Natl Acad Sci USA; 101:8174-9.

11. Grant I, Gonzalez R, Carey CL, Natarajan L, Wolfson T. (2003). Non-acute (residual) neurocognitive effects of cannabis use: a meta-analytic study. J Int Neuropsychol Soc; 9:679-689. 
12. Grant I, Atkinson HJ, Gouaux B, Wilsey B. (2012), Medical marijuana: clearing away the smoke. Open Neurol J; 6:18-25.

13. Gugliotta, M., Bisiacchi, P. S., Cendron, M., Tressoldi, P. E., \&Vio, C. (2009). BVN 12-18-Batteria per la ValutazioneNeuropsicologica per l'adolescenza.Edizioni Erickson, Gardolo.

14. Hindocha C, Freeman TP, Xia JX, Shaban NDC, \& Curran HV (2017). Acute memory and psychotomimetic effects of cannabis and tobacco both 'joint' and individually: a placebo-controlled trial. Psychol Med, 47(15), 2708-2719. doi: 10.1017/S0033291717001222.

15. HoaT.Vo, Rebecca Schacht, Miriam Mintzer\& Marc Fishman (2014), Working Memory Impairment in Cannabis- and Opioid-Dependent Adolescents, Substance Abuse, 35:4, 387-390, DOI: 10.1080/08897077.2014.954027.

16. Ilan AB, Smith ME, \&Gevins A (2004). Effects of marijuana on neurophysiological signals of working and episodic memory. Psychopharmacology (Berl), 176(2), 214-222. doi: 10.1007/s00213-004-1868-9.

17. Lisdahl K. M. \& Price J. S. (2012), Increased marijuana use and gender predict poorer cognitive functioning in adolescents and emerging adults.Journal of the International Neuropsychological Society, 18(4), 678-688. doi:10.1017/ S1355617712000276.

18. Lundqvist $\mathrm{T}$ (2005). Cognitive consequences of cannabis use: comparison with abuse of stimulants and heroin with regard to attention, memory and executive functions. PharmacolBiochemBehav, 81(2), 319-330. doi:10.1016/j.pbb.2005.02.017.

19. Meier RH, Caspi A, Ambler A, et al. (2012). Persistent cannabis users show neuropscychological decline from childhood to midlife. Proc Natl Acad Sci U S A;109:ES657-ES664.

20. Millsaps CL, Azrin RL, Mittenberg W. (1994), Neuropsychological effects of chronic cannabis use on the memory and intelligence of adolescents. J Child Adolescent Subst Abuse; 3: 47-55.

21. Morin J. G., Afzali M. H., Bourque J., Stewart S. H., Séguin J. R., O'Leary-Barrett M., Conrod, P. J. (2018), A Population-Based Analysis of the Relationship Between Substance Use and Adolescent Cognitive Development. American Journal of Psychiatry; appi.ajp.2018.1 DOI:10.1176/ appi.ajp.18020202.

22. O'Shea M., Singh ME., McGregor IS., Mallet PE. (2004), Chronic cannabinoid exposure produces lasting memory impairment and increased anxiety in adolescent but not adult rats. J Psychopharmacol 18: 502-508.

23. O'Shea M., McGregor IS., Mallet PE. (2006), Repeated cannabinoid exposure during perinatal, adolescent or early adult ages produces similar longlasting deficits in object recognition and reduced social interaction in rats. J Psychopharmacol 20: 611-621.

24. Orsini A., Pezzuti L. \&Picone L. (2012), WISC-IV: ContributoallataraturaItaliana (WISC-IV Italian) ed. Florence, Italy: Giunti O. S.

25. Pope H. G. J. \&Yurgelun-Todd D. (1996), The residual cognitive effects of heavy marijuana use in college students. JAMA, 275(7), 521-527.

26. Pope HG Jr, Gruber AJ, Yurgelun-Todd D. (2001). Residual neuropsychologic effects of cannabis. Curr Psychiatry Rep; 3:507 - 12.

27. Pope HG Jr. (2002). Cannabis, cognition and residual confounding. J Am Med Ass; 287:1172 - 4.

28. Porath-Waller A.J. (2009), Clearing the Smoke on Cannabis. Chronic Use and Cognitive Functioning and Mental Health. Canadian Centre on Substance Abuse.

29. Public Health Agency of Canada (2018). Preventing Problematic Substance Use in Youth. Available online at:

https://www.canada.ca/en/public-health/news/2018/10/preventing-problematic-substance-use-in-youth.ht ml. Accessed May 12, 2019.

30. Rubino T., Vigano' D., Realini N., Guidali C., Braida D., Capurro V., Castiglioni C., Cherubino F., Romualdi P., Candeletti S., Sala M., Parolaro D. (2008), Chronic delta (9) -tetrahydrocannabinol during adolescence provokes sex-dependent changes in the emotional profile in adult rats: behavioral and biochemical correlates. Neuropsychopharmacology.;33(11):2760-71.

31. Rubino T., Zamberletti E., Parolaro D. (2011), Uso di cannabis in adolescenza come fattore di rischio per le malattiepsichiatriche e la dipendenza da altredroghe. In "Cannabis e dannialla salute". 
32. Solowij N, Stephens RS, Roffman RA, Babor T, Kadden R, Miller M, ... Group, M. T. P. R. (2002). Cognitive functioning of long-term heavy cannabis users seeking treatment. JAMA, 287(9), 1123-1131.

33. Solowij N, Battisti R. (2008). The chronic effects of cannabis on memory in humans: a review. Curr Drug Abuse Rev; 1:81-98.

34. Ullman H, Almeida R, Klingberg T. (2014). Structure maturation and brain activity predict future working memory capacity during childhood development. J Neurosci; 29:34:1592-1599.

35. Volkow N. D., Swanson J. M., Evins A. E., DeLisi L. E., Meier M. H., Gonzalez R., et al. (2016). Effects of cannabis use on human behavior, including cognition, motivation, and psychosis: a review. JAMA Psychiatry 73, 292-297. 10.1001/jamapsychiatry.2015.3278. 